\title{
Some functors arising from the consideration of torsion theories over noncommutative rings
}

\section{Jonathan S. Golan}

\begin{abstract}
To each associative (but not necessarily commutative) ring $R$ we assign the complete distributive lattice $R$-tors of (hereditary) torsion theories over $R$-mod . We consider two ways of making this process functorial - once contravariantly and once covariantly - by selecting appropriate subcategories of the category of associative rings. Combined with a functor due to Rota, this gives us functors from these subcategories to the category of commutative rings.
\end{abstract}

Let $R$ be an associative (but not necessarily commutative) ring with unit element 1 . We denote by $R$-mod the category of all unitary left $R$-modules and by $R$-tors the complete distributive lattice of all (hereditary) torsion theories over $R-\bmod$. See [4] for details. If $\tau \in R$-tors and if $M$ is a left $R$-module, then $T_{\tau}(M)$ will denote the T-torsion submodule of $M$ and $Q_{\tau}(M)$ will denote the localization of $M$ at $\tau$. The ring of quotients of $R$ at $\tau$ will be denoted by $R_{\tau}$. For each left $R$-module $M$, we have a homomorphism $\hat{\tau}_{M}: M \rightarrow Q_{\tau}(M)$ of left $R$-modules. Also, we have a ring homomorphism $\hat{\tau}: R \rightarrow R_{\tau}$. If this ring homomorphism is a left flat ring epimorphism (that is, if $\hat{\tau}$ is an epimorphism in the category of rings and if $R_{\tau}$ is flat as a pight $R$-module) then the torsion theory $\tau$ is said to be perfect. Left flat

Received 3 August 1976. 
ring epimorphisms are studied in some detail in [9]. It is well-known that every left flat ring epimorphism is in fact of the form $\hat{\tau}$ for some perfect torsion theory $\tau$. Moreover, $\tau \in R$-tors is perfect if and only if $Q_{\tau}(M)=R_{\tau} \otimes_{R} M$ for every left $R$-module $M$. If $\gamma: R \rightarrow S$ is a ring homomorphism then $\gamma$ defines a function $\gamma_{\#}: R$-tors $\rightarrow S$-tors which assigns to $\tau \in R$-tors that torsion theory $\sigma \in S$-tors characterized by the property that a left $S$-module $N$ is $\sigma$-torsion if and only if it is $\tau$-torsion as a left $R$-module. If $S$ is flat as a right $R$-module, then $\gamma$ also defines a function $\gamma^{\#}: S$-tors $\rightarrow R$-tors which assigns to $\sigma \in S$-tors that torsion theory $\tau \in R$-tors characterized by the property that a left $R$-module $M$ is $\tau$-torsion if and only if $S \otimes_{R} M$ is $\sigma$-torsion. If $\gamma: R \rightarrow S$ is a left flat ring epimorphism, say $\gamma=\hat{\tau}$ for a perfect torsion theory $\tau \in R$-tors, then $\gamma_{\#}$ and $\gamma^{\#}$ induce a bijection between $S$-tors and $\left\{\tau^{\prime} \in R\right.$-tors $\left.\mid \tau^{\prime} \geq \tau\right\}$. See Proposition 17.14 of [4]. Thus we have a contravariant functor $F$ from the category of rings and left flat ring epimorphisms to the category of complete lattices given by $F(R)=R$-tors and $F(\gamma)=\gamma^{\#}$.

We now seek another subcategory of the category of rings from which we can construct a covariant functor to the category of complete lattices which behaves on objects in the same way as $F$, namely which assigns to each ring $R$ the complete lattice $R$-tors. For this we need some more definitions and remarks.

If $\gamma: R \rightarrow S$ is a ring homomorphism and if $\tau \in R$-tors, it is not necessarily true that a left $S$-module is $\gamma_{\#}(\tau)$-torsionfree if and only if it is $\tau$-torsionfree as a left $R$-module. When this condition does hold, we say that the torsion theory $\tau$ is compatible with $\gamma$. The notion of compatibility between a torsion theory and a ring homomorphism has recently been studied by Louden [5], who also provides several examples of this phenomenon. Proposition 9.8 of [4] asserts that every torsion theory is compatible with a ring surjection; Proposition 17.15 of [4] asserts that if $\tau \in R$-tors is perfect and if $\tau^{\prime} \geq \tau$ then $\tau^{\prime}$ is compatible with $\hat{\tau}$. A torsion theory $\tau \in R$-tors is stable if and only if the class of all $\tau$-torsion left $R$-modules is closed under taking essential extensions. 
Stable torsion theories are very important in the theory of noncomutative localization; they were first introduced and characterized in [2]. The Goldie torsion theory is a common example of a stable torsion theory. A ring $R$ is left stable if and only if every member of $R$-tors is stable. Such rings have been studied in $[1,6,7]$; commutative noetherian rings are left stable and left stability is a Morita invariant.

In [4] we showed that the lattice $R$-tors is always distributive. Raynaud has extended this result to show that $R$-tors in fact always satisfies the join-infinite distributive identity, namely that for every $\tau \in R$-tors and every $U \subseteq R$-tors, we have

$$
\tau \wedge(\vee U)=\vee\left\{\tau \wedge \tau^{\prime} \mid \tau^{\prime} \in U\right\} \text {. }
$$

Thus the lattice $R$-tors is brouwerian [8]. In a private conversation, $D$. Strauss has pointed out that if $R$ is left stable then the dual of this, namely the meet-infinite distributive identity also holds. Thus we have:

PROPOSITION 1. If $R$ is a left stable ring then for every $\tau \in R$-tors and every $U \subseteq R$-tors we have $\tau \vee(\Lambda U)=\wedge\left\{\tau \vee \tau^{\prime} \mid \tau^{\prime} \in U\right\}$.

Proof. For each $\tau^{\prime} \in U$ we have $\tau^{\prime} \geq \Lambda U$ and so $\tau \vee \tau^{\prime} \geq \tau \vee(\Lambda U)$. If $\tau^{\prime \prime}=\Lambda\left\{\tau \vee \tau^{\prime} \mid \tau^{\prime} \in U\right\}$ we then have that $\tau^{\prime \prime} \geq \tau \vee(\Lambda U)$. Now assume that this inequality is strict. Then there exists a nonzero left $R$-module $M$ which is $\tau^{\prime \prime}$-torsion but not $[\tau \vee(\Lambda U)]$-torsion. Indeed, replacing $M$ by $M / T \tau \vee(\Lambda U)(M)$, we can assume that $M$ is $[\tau \vee(\Lambda U)]$-torsionfree and hence that it is both $\tau$-torsionfree and $(N U)$-torsionfree, as well as being $\tau^{\prime \prime}$-torsion. Since $\tau^{\prime \prime}$ is stable, the injective hull $E(M)$ of $M$ also has these properties. In particular, if $\tau^{\prime} \in U$ then $E(M)$ cannot be $\tau^{\prime}$-torsionfree. By [4, Proposition 11.2], there then exists a submodule $N$ of $E(M)$ such that $E(M)=N \oplus T_{\tau^{\prime}}(E(M))$. This implies that $N$ is both $\tau$-torsionfree and $\tau^{\prime}$-torsionfree and so is ( $\left.\tau \vee \tau^{\prime}\right)$ torsionfree, a contradiction unless $N=0$. Thus $E(M)$ must be $\tau^{\prime}-$ torsion for every $\tau^{\prime} \in U$. This implies that $E(M)$ is $(\Lambda U)$-torsion, which is also a contradiction.

Another interesting aspect of stability is the following.

PROPOSITION 2. Let $\tau \in R$-tors be perfect and let $\tau^{\prime} \in R$-tors be stable. Then $\hat{\tau}_{\#}\left(\tau \vee \tau^{\prime}\right)=\hat{\tau}_{\#}\left(\tau^{\prime}\right)$. 
Proof. By [4, Proposition 17.14] there exists a torsion theory $\tau^{\prime \prime} \geq \tau$ satisfying the condition that $\hat{\tau}_{\#}\left(\tau^{\prime \prime}\right)=\hat{\tau}_{\#}\left(\tau^{\prime}\right)$. Indeed, $\tau^{\prime \prime}$ is characterized by the property that a left $R$-module $M$ is $\tau^{\prime \prime}$-torsion if and only if $Q_{\tau}(M)$ is $\hat{\tau}_{\#}\left(\tau^{\prime}\right)$-torsion as a left $R_{\tau}$-module. We therefore want to show that $\tau^{\prime \prime}=\tau \vee \tau^{\prime}$. Let $M$ be a $\left(\tau \vee \tau^{\prime}\right)$-torsionfree left $R$-module. Then $M$ is $\tau$-torsionfree and so the homomorphism $\hat{\tau}_{M}: M \rightarrow Q_{\tau}(M)$ is an essential extension, implying that $Q_{\tau}(M)$ is $\tau^{\prime}-$ torsionfree as a left $R$-module. Therefore $Q_{\tau}(M)$ is $\hat{\tau}_{\#}\left(\tau^{\prime}\right)$-torsionfree as a left $R_{\tau}$-module and so $M$ is $\tau^{\prime \prime}$-torsionfree since $\tau^{\prime \prime}$ is compatible with $\hat{\tau}$. This proves that $\tau \vee \tau^{\prime} \geq \tau^{\prime \prime}$. Assume that this inequality is strict. Then by [4, Proposition 17.14] we have that $\hat{\tau}_{\#}\left(\tau \vee \tau^{\prime}\right)>\hat{\tau}_{\#}\left(\tau^{\prime \prime}\right)=\hat{\tau}_{\#}\left(\tau^{\prime}\right)$ and so there exists a left $R_{\tau}$-module $N$ which is $\hat{\tau}_{\#}\left(\tau \vee \tau^{\prime}\right)$-torsion but not $\hat{\tau}_{\#}\left(\tau^{\prime}\right)$-torsion. Indeed, by factoring out the $\hat{\tau}_{\#}\left(\tau^{\prime}\right)$-torsion submodule of $N$ we can assume that $N$ is $\hat{\tau}_{\#}\left(\tau^{\prime}\right)-$ torsionfree. Since $N$ is a left $R_{\tau}$-module and since $\tau$ is perfect, we know that $N$ is $\tau$-torsionfree as a left $R$-module. Since $N$ is $\hat{\tau}_{\#}\left(\tau \vee \tau^{\prime}\right)$-torsion, it is $\left(\tau \vee \tau^{\prime}\right)$-torsion as a left $R$-module and so cannot be $\tau^{\prime}$-torsionfree as a left $R$-module. Let $M$ be the $\tau^{\prime}$-torsion submodule of $N$. Since $M$ is also $\tau$-torsionfree, the homomorphism $\hat{\tau}_{M}$ is an essential extension and so $Q_{\tau}(M)$ is also $\tau^{\prime}$-torsion by the stability of $\tau^{\prime}$. Therefore $Q_{\tau}(M)$ is $\hat{\tau}_{\#}\left(\tau^{\prime}\right)$-torsion as a left $R_{\tau}$-module. But $Q_{\tau}(M)$ is an $R_{\tau}$-submodule of the $\hat{\tau}_{\#}\left(\tau^{\prime}\right)$-torsionfree left $R$-module $N$, which implies that we must have $M=0$, a contradiction. This proves that $\tau \vee \tau^{\prime}=\tau^{\prime \prime}$.

As a consequence of this result we obtain the following proposition.

PROPOSITION 3. If $R$ is a left stable ring and if $\tau \in R$-tors is perfect then $\hat{\tau}_{\#}: R$-tors $\rightarrow R_{\tau}$-tors is a morphism of complete lattices.

Proof. It is an immediate consequence of the definitions that $\hat{\tau}_{\#}$ is a morphism of complete meet semilattices. If $U$ is a subset of $R$-tors and if $N$ is a left $R_{\tau}$-module, then, by Proposition 2, we know that $N$ 
is $\hat{\tau}_{\#}(V U)$-torsionfree if and only if $N$ is $\hat{\tau}_{\#}[(V U) \vee \tau]$-torsionfree. Since $(v U) \vee \tau$ is compatible with $\hat{\tau}$, this holds if and only if $N$ is $[(v U) \vee \tau]$-torsionfree when considered as a left $R$-module; that is, it holds if and only if $N$ is $\left(\tau^{\prime} \vee \tau\right)$-torsionfree for every $\tau^{\prime} \in U$. Since $\tau^{\prime} \vee \tau$ is compatible with $\hat{\tau}$, this holds if and only if $N$ is $\hat{\tau}_{\#}\left(\tau^{\prime} \vee \tau\right)$-torsionfree as a left $R_{\tau}$-module for every $\tau^{\prime} \in U$ and again by Proposition 2 this holds if and only if $N$ is $\hat{\tau}_{\#}\left(\tau^{\prime}\right)$-torsionfree for every $\tau^{\prime} \in U$ or, in other words, if and only if $N$ is torsionfree with respect to $v\left\{\hat{\tau}_{\#}\left(\tau^{\prime}\right) \mid \tau^{\prime} \in U\right\}$.

Thus we now have a covariant functor $G$ from the category of left stable rings and left flat ring epimorphisms to the category of complete lattices and morphisms of complete lattices given by $G(R)=R$-tors and $G(\gamma)=\gamma_{\#}$.

In conclusion, let us note one possible use of the functors $F$ and $G$. Rota has defined a "valuation" functor $V(-)$ from the category of distributive lattices and lattice homomorphisms to the category of commutative rings and ring homomorphisms. See [3] for details. If we combine this functor with the functor $F$ [respectively the functor $G$ ] defined above we obtain a contravariant [respectively covariant] functor from the category of [left stable] rings and left flat ring epimorphisms to the category of commutative rings and ring homomorphisms.

\section{References}

[1] Ladislav Bican and Tomáš Kepka, "On stable rings", Pubz. Math. Debrecen 22 (1975), 235-244.

[2] Pierre Gabriel, "Des catégories abéliennes", Bull. Soc. Math. France $90(1962), 323-448$.

[3] Ladnor Geissinger, "Valuations on distributive lattices I", Arch. Math. (Basel) 24 (1973), 230-239.

[4] Jonathan S. Golan, Localization of noncommutative rings (Marcel Dekker, New York, 1975). 
[5] Kenneth Louden, "Torsion theories and ring extensions", Comm. Algebra 4 (1976), 503-532.

[6] Zoltan Papp, "On stable noetherian rings", Trans. Amer. Math. Soc. 213 (1975), 107-114.

[7] Zoltan Papp, "Semi-stability and topologies on R-sp ", preprint 1975.

[8] Jacques Raynaud, "Localisations et topologie de Stone", preprint 1975.

[9] L. Silver, "Noncommutative localizations and applications", J. Algebra 7 (1967), 44-76.

Department of Mathematics,

University of Haifa,

Mount Carmel,

Haifa,

Israel. 